\title{
Computer-Assisted Inverse Design of Inorganic Electrides
}

\author{
Yunwei Zhang, ${ }^{1,2}$ Hui Wang, ${ }^{1}$ Yanchao Wang, ${ }^{1}$ Lijun Zhang, ${ }^{3,1}$ and Yanming Ma ${ }^{1,2, *}$ \\ ${ }^{1}$ State Key Lab of Superhard Materials, Jilin University, Changchun 130012, China \\ ${ }^{2}$ Beijing Computational Science Research Center, Beijing 100084, China \\ ${ }^{3}$ Key Laboratory of Automobile Materials of MOE and Department of Materials Science, \\ Jilin University, Changchun 130012, China
}

(Received 25 July 2016; revised manuscript received 17 November 2016; published 14 February 2017; corrected 23 February 2017)

\begin{abstract}
Electrides are intrinsic electron-rich materials enabling applications as excellent electron emitters, superior catalysts, and strong reducing agents. There are a number of organic electrides; however, their instability at room temperature and sensitivity to moisture are bottlenecks for their practical uses. Known inorganic electrides are rare, but they appear to have greater thermal stability at ambient conditions and are thus better characterized for application. Here, we develop a computer-assisted inverse-design method for searching for a large variety of inorganic electrides unbiased by any known electride structures. It uses the intrinsic property of interstitial electron localization of electrides as the global variable function for swarm intelligence structure searches. We construct two rules of thumb on the design of inorganic electrides pointing to electron-rich ionic systems and low electronegativity of the cationic elements involved. By screening 99 such binary compounds through large-scale computer simulations, we identify 24 stable and 65 metastable new inorganic electrides that show distinct three-, two-, and zero-dimensional conductive properties, among which 18 are existing compounds that have not been pointed to as electrides. Our work reveals the rich abundance of inorganic electrides by providing 33 hitherto unexpected structure prototypes of electrides, of which 19 are not in the known structure databases.
\end{abstract}

DOI: 10.1103/PhysRevX.7.011017

\section{INTRODUCTION}

Electrides are ionic solids that consist of a cationic framework with intrinsic excess electrons trapped in the lattice voids playing the role of anions [1,2]. The localized electrons make electrides relate to salts with $F$ centers, but markedly differ from metals containing delocalized electrons [3]. Early synthesized electrides have been organic species [4-6] consisting of cationic alkali metals ions complexed with crown ethers. The solvent electrons released by the alkali metals are trapped in potential wells between these complexed cations. Although organic electrides are good low-temperature electron emitters and strong reducing agents [7-9], their thermal instability above $-40{ }^{\circ} \mathrm{C}$ and sensitivity to air and water restrict their practical application.

The inorganic electride $\mathrm{C} 12 \mathrm{~A} 7: e^{-}$that is thermally and chemically stable at ambient conditions was synthesized by removing oxygen ions from the center of clathrate $\mathrm{Ca}-\mathrm{Al}-\mathrm{O}$ cages in mayenite $\left(12 \mathrm{CaO}_{7} \cdot \mathrm{Al}_{2} \mathrm{O}_{3}\right)$ [10]. It is even reported to be air stable at a high temperature of $300{ }^{\circ} \mathrm{C}$.

\footnotetext{
*mym@calypso.cn, mym@jlu.edu.cn
}

Published by the American Physical Society under the terms of the Creative Commons Attribution 3.0 License. Further distribution of this work must maintain attribution to the author(s) and the published article's title, journal citation, and DOI.
Subject Areas: Chemical Physics, Computational Physics, Materials Science

More strikingly, it shows excellent field-emission properties with a lower work function than carbon nanotubes [11] and exhibits catalytic activity an order of magnitude higher than conventional catalysts, such as $\mathrm{Fe}-\mathrm{Al}_{2} \mathrm{O}_{3}-\mathrm{K}_{2} \mathrm{O}$ [12], for ammonia synthesis when loaded on $\mathrm{Ru}$ [13].

$\mathrm{Ca}_{2} \mathrm{~N}$ is a two-dimensional electride in which electrons are weakly localized in the $2 \mathrm{D}$ interspaces between the two positively charged $\left[\mathrm{Ca}_{2} \mathrm{~N}\right]^{+}$layers [14]. $\mathrm{Ca}_{2} \mathrm{~N}$ was used as an efficient electron donating agent in the transfer hydrogenation of alkynes and alkenes [15]. The predicted electron transport properties of monolayer $\mathrm{Ca}_{2} \mathrm{~N}$ encapsulated by graphene are excellent if compared with typical 2D electronic systems (e.g., GaAs-AlGaAs heterojunction, $\mathrm{LaAlO}_{3}-\mathrm{SrTiO}_{3}$ interface, and graphene) [16].

High-pressure electrides, as an important category of inorganic electrides, were predicted in various simple elemental metals [17-21]. These elemental electrides are unique, and differ from electron-rich ionic electrides observed at ambient pressure [22,23]. However, these high-pressure electrides are not quenchable to ambient conditions, and are unlikely to be practically useful.

A substitutional screening calculation has been conducted to search for $2 \mathrm{D}$ electrides $[24,25]$. The anti- $\mathrm{CdCl}_{2}$ structure of $\mathrm{Ca}_{2} \mathrm{~N}$ was used as a prototype electride structure where $\mathrm{Ca}$ and $\mathrm{N}$ were replaced with alternative metal elements (e.g., some alkali and transition metals) and nonmetal elements (e.g., halogens, $\mathrm{O}$ and $\mathrm{C}$ ), respectively. 
The method was particularly successful when the potential electrides were crystalized in the same structure, and a predicted $\mathrm{Y}_{2} \mathrm{C}$ electride has been observed experimentally [26]. However, this method is limited because the predicted electrides rely heavily on known structures, and, thus, finding electrides with unknown structures is unlikely.

Inorganic electrides show greater stability but are rarely known under ambient conditions [10,26-28], as described, limiting their applicability, and their computer-assisted design is highly desirable. In this study, we develop an efficient inverse-design method that relies on the intrinsic physical properties of interstitial electron localization instead of known electride structures to search for new, diverse inorganic electrides. This method is in contrast to the traditional structure-searching algorithm where the energy has been used as the searching criterion. Obtaining electride structures with intrinsic property of electron localization is our main concern. Local geometric optimizations are performed for structures generated from the structure searches. Our method is incorporated into our CALYPSO structure prediction code [29,30], enabling automatic structure searches through intelligent exploration of unknown structure types.

Our method reliably identifies the known electride structures in $\mathrm{Ca}_{2} \mathrm{~N}$ [14] and transparent dense $\mathrm{Na}$ [17] by using the input information of chemical compositions alone. Through extensive inverse-design simulations on 99 binary ionic systems, 89 inorganic electrides are newly designed, of which 18 have already been synthesized but are yet to be identified as electrides. Among the other 71 designed electrides waiting for experimental syntheses, 18 are energetically stable, whereas 53 are metastable. We unravel the rich abundance of inorganic electrides by providing 33 new prototype structures that are hitherto unexpected.

\section{RESULTS}

\section{A. Inverse-design method}

Our inverse-design method is developed on top of our swarm intelligence CALYPSO structure searching method that can explore structures intelligently by using the chemical composition alone for a compound without relying on known structural information [30]. The current implementation uses the interstitial electron localization rather than the total energy as the global variable function. This is consistent with the strong electron localization in the interstitials of lattice being the intrinsic physical property of electrides. Our structural design follows the principle of inverse design, where the structure searches identify structures with a desirable functionality for a given chemical system. Our previous inverse-design exercise has been applied to the searching of superhard materials [31]. The electron localization function (ELF) [32-34], which identifies core, binding, and lone-pair regions in chemical systems, provides a semiquantitative index of interstitial electron localization and has been used as a powerful tool for analyzing electrides $[17,19,35,36]$. Although the ELF values able to characterize good electron localization depend on specific systems, ELF values larger than 0.75 described electron localization well for most systems [37,38].

Here, we define the degree of interstitial electron localization $\omega=V_{\text {inter }} / V_{\text {cry }}$ as the searching criterion, where $V_{\text {inter }}$ and $V_{\text {cry }}$ are the volumes of the interstitial regions where electrons are exactly localized and the simulation cell, respectively. $V_{\text {inter }}$ can be critically determined from the reliable ELF calculation. By making use of the ELF map (with isosurface of 0.75), we differentiate the regions for interstitial localized electrons from other types of localized electrons, such as core electrons around ions and electrons shared by covalent bonds. Taking an electride structure of $\mathrm{Sr}_{2} \mathrm{C}$ as an illustration [Fig. 1(a)], we show how to obtain a precise $V_{\text {inter }}$ for interstitially localized electrons, after excluding the ionic cores areas [top panel, Fig. 1(a)] and the covalent bonding areas [middle panel, Fig. 1(a)], where the electron density maximum locates in the lattice. The discarded regions for the ionic cores area are shown as pink spheres [top panel, Fig. 1(a)] with radii $R_{\text {cut }}$ as derived from explicitly calculated ELF maps for various binary compounds, as listed in Fig. S1(a) of the Supplemental Material [39]. Note that the $R_{\text {cut }}$ is typically calculated to be larger than the Pauling ionic radii [40] of elements in order to give a clean removal of electrons sitting at ionic cores. The covalent bonds are determined by direct calculations of interatomic distances (i.e., covalent bond lengths) between (a)

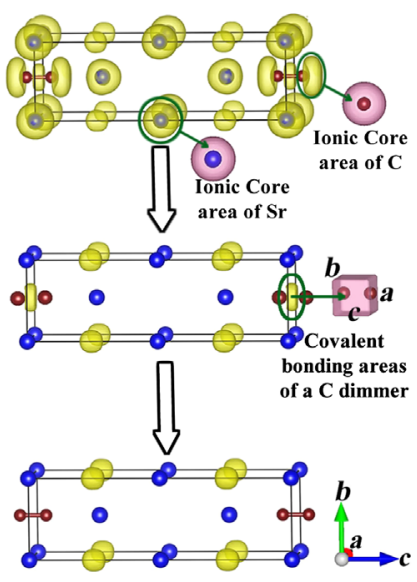

(b)

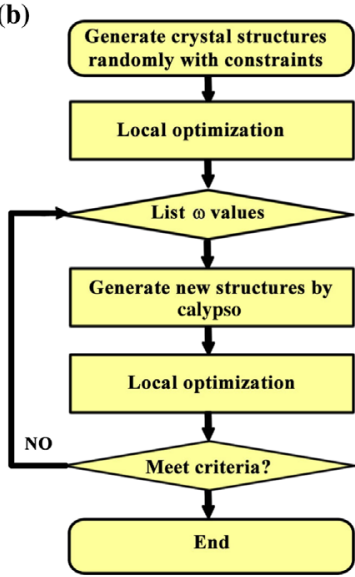

FIG. 1. Computational details of calculation of $\omega$ and inversedesign scheme for electrides. (a) A step-by-step process of calculating $V_{\text {inter }}$ in a tetragonal $\mathrm{Sr}_{2} \mathrm{C}$ crystal. In the ELF map with an isosurface of 0.75 , the regions for localized electrons are shown as yellow. Exclusion of ionic cores areas for localized electrons from the crystal as illustrated by $\mathrm{Sr}$ and $\mathrm{C}$ atoms (top panel). Exclusion of $\mathrm{C}-\mathrm{C}$ covalent bonding areas from the crystal (middle panel). The volume of the interstitial areas where the electrons are localized ( $V_{\text {inter }}$, shown as yellow in ELF map in bottom panel). (b) Flow chart of the CALYPSO module for searching for electrides with the inverse-design method. 
anionic atoms. Figure S1(b) of the Supplemental Material [39] lists the maximum bond lengths allowed in our simulations as a critical length for forming covalent bonds at ambient condition. Once the covalent bonds are determined, the covalent bonding areas are defined as cuboids [pink in the middle panel or Fig. 1(a)], constructed with the parameters of $a=b=R_{\text {cut }}$, and $c$ equals the covalent bond length in the crystal. After excluding the ionic cores areas and the covalent bonding areas, we can eventually obtain the $V_{\text {inter }}$ for interstitial localized electrons, i.e., the volumes of regions [yellow in the bottom panel or Fig. 1(a)] where ELF values are at and above 0.75 . The higher $\omega$ represents that there exist larger areas for interstitial localized electrons and a stronger potential for a structure to be an electride. The discarded regions are depicted as pink spheres with $R_{\text {cut }}$ of $1.6 \AA$ for $\mathrm{Sr}$ and $1.5 \AA$ for $\mathrm{C}$ [see $R_{\text {cut }}$ in Fig. S1(a) [39]], respectively. Note that $R_{\text {cut }}$ is typically calculated to be larger than the Pauling ionic radii of elements in order to give a clean removal of electrons sitting at ionic cores. The discarded covalent bonding areas for localized electrons are defined as pink cuboids in our simulation in which $a=b=1.6 \AA$ ( $R_{\text {cut }}$ of $\left.\mathrm{Sr}\right)$ and $c=1.31 \AA$ (the $\mathrm{C}-\mathrm{C}$ bond length within the $\mathrm{C}$ dimers). In practice, we deal with the ELFCAR (the output file of the
ELF calculation in the VASP program) file, where a threedimensional $36 \times 36 \times 120(=15520)$ grid is used to simulate $V_{\text {inter }}$ of the $\mathrm{Sr}_{2} \mathrm{C}$ crystal where ELF values are calculated for every grid point. $V_{\text {inter }}$ is derived from the number of grid points where ELF values are larger than 0.75 in the interstitial regions (it is 1006 for $\mathrm{Sr}_{2} \mathrm{C}$ ). Then, the $\omega$ value of $\mathrm{Sr}_{2} \mathrm{C}$ is calculated to be $V_{\text {inter }} / V_{\text {cry }}$; i.e., $1006 / 15520=0.65 \%$.

Our inverse-design approach is outlined in Fig. 1(b). In the first generation, structures are randomly generated by symmetry constraints within 230 space groups. Once a particular space group is selected, the lattice parameters are then obtained within the chosen symmetry. The corresponding atomic coordinates are generated by the crystallographic symmetry operations [29]. Structures are then geometrically optimized for seeking their local minima in the potential surface. The $\omega$ values of the optimized structures are evaluated to rank good electride structures. A certain number of structures (here, we choose $60 \%$ of a population) with high $\omega$ values are evolved into the next generation by swarm optimization via smart learning of the personal and global best electride structures [29,41]. The other $40 \%$ of structures in each generation are randomly generated to increase structural diversity during the (a)
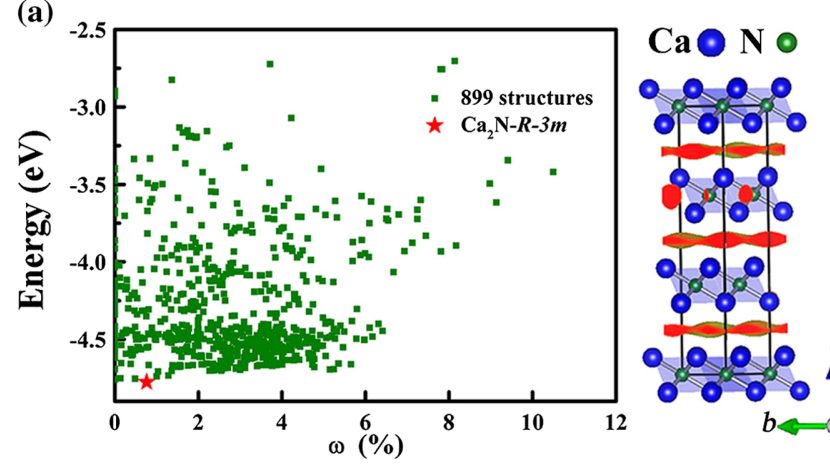

(b)
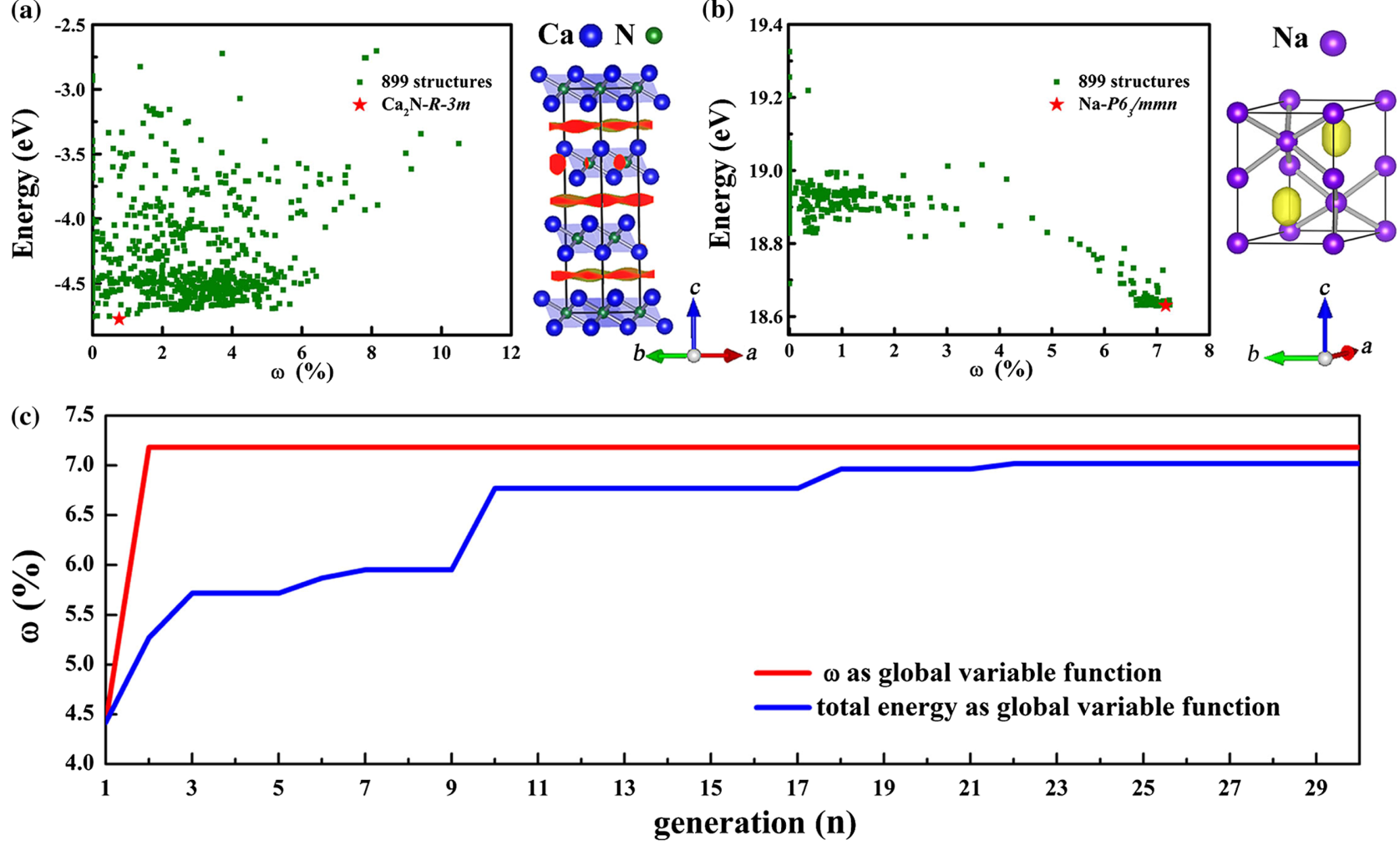

FIG. 2. Benchmarking to two known electrides: $\mathrm{Ca}_{2} \mathrm{~N}$ at ambient pressure and transparent $\mathrm{Na}$ at 320 GPa. $\omega$ versus energy maps of $\mathrm{Ca}_{2} \mathrm{~N}$ at ambient pressure (a) and transparent $\mathrm{Na}$ at $320 \mathrm{GPa}$ (b). Symbols represent structures produced by the CALYPSO run within 30 generations. The experimental anti-CdCl${ }_{2}$ and $h P 4$ electride structures for $\mathrm{Ca}_{2} \mathrm{~N}$ and $\mathrm{Na}$ are shown as red stars in the maps. (c) Evolution of the optimum $\omega$ versus generations for $\mathrm{Ca}_{2} \mathrm{~N}$ using $\omega$ (red line) and total energy (blue line) as global variable functions, respectively. The $x$ axis is the $n$th generation in an inverse-design simulation by CALYPSO. 
structure evolution. A symmetrization procedure is applied in order to refine the resultant electride structures. Eventually, we construct a $\omega$ versus energy map to trace the structures generated and identify the electrides [Figs. 2(a) and 2(b)].

\section{B. Benchmarkings to known electrides}

We benchmark our inverse-design method to two known electride systems: $\mathrm{Ca}_{2} \mathrm{~N}$ (its variants, such as $\mathrm{Sr}_{2} \mathrm{~N}$ and $\mathrm{Y}_{2} \mathrm{C}$, are shown in Fig. S3 of the Supplemental Material [39]) at ambient pressure and the high-pressure electride, transparent $\mathrm{Na}$ at $320 \mathrm{GPa}$. With the sole input information of the chemical composition of $\mathrm{Ca}: \mathrm{N} 2: 1$, our calculations readily identify the experimental anti- $\mathrm{CdCl}_{2}$ electride structure of $\mathrm{Ca}_{2} \mathrm{~N}$ [right-hand panel, Fig. 2(a)] via the calculated $\omega$ versus energy map [left-hand panel, Fig. 2(a)]. Our calculations also correctly reproduce the $h P 4$ electride structure [right-hand panel, Fig. 2(b)] of $\mathrm{Na}$ at $320 \mathrm{GPa}$ in the calculated $\omega$ versus energy map [left-hand panel, Fig. 2(b)]. Thus, our method is also suitable for designing high-pressure electrides. For these benchmarking systems, it is found that the same electride structure can be obtained in different runs of the inverse-design simulations, which demonstrates the repeatability and reliability of our method (see Fig. S4 of Supplemental Material [39]).

We find that using $\omega$ as the global variable function, rather than the energy, increases the efficiency of the optimum electride structure search. As an illustration, we compare the structure search results for the $\mathrm{Ca}_{2} \mathrm{~N}$ system using $\omega$ and total energy as global variable functions, respectively. During these two searches, $100 \%$ of structures are evolved into the next generation by the swarm optimization operation without introducing any random structures. The evolution of $\omega$ values with generation for each method is shown in Fig. 2(c). Of the 900 structures produced in 30 generations, the optimum electride structure with the largest $\omega$ value $7.2 \%$ is obtained in the second generation in the current method; however, the largest $\omega$ value $7.0 \%$ appears only in the 22 nd generation using total energy as the variable. This demonstrates the viable efficiency of our inverse-design method for searching for optimum electrides over the traditional method. Furthermore, the efficiency of our method is found to be independent of the systems chosen, as has been demonstrated in the $\mathrm{La}_{2} \mathrm{C}$ system (see Fig. S5 of Supplemental Material [39]). It is noteworthy that, compared to the traditional searching method, our inverse-design scheme runs the risk of missing the global stable structure, however, only in the case that the structure is not an electride. If a material has the fate to be an electride, our method can identify it. The energetic stability of our inversely designed electrides has been confirmed by the energy-based structure searching simulations (see Fig. S6 of Supplemental Material [39]).

\section{Design principles for inorganic electrides}

Electrides, such as C12A7: $e^{-}$and $\left[M_{2} N\right]^{+} \cdot\left(e^{-}\right)$ $(M=\mathrm{Ca}, \mathrm{Sr}$, and $\mathrm{Ba})$, contain an intrinsic excess of electrons trapped in vacancies acting as anions to balance the positive charges of the lattice keeping the electroneutrality of systems. Excess electrons in a chemical system are a necessary condition for stabilizing an electride; therefore, this might be used as a design principle in searching for inorganic electrides. We prove this design principle (no. 1 rule of thumb) in a model system $\left[\mathrm{Ca}_{2} \mathrm{~N}\right]^{+}$ by removing one excess electron per formula unit of $\mathrm{Ca}_{2} \mathrm{~N}$. By doing so, we define in our density-functional theory (DFT) calculation the $\left[\mathrm{Ca}_{2} \mathrm{~N}\right]^{+}$system with one electron less by setting the control parameter of NELECT to 8 in the VASP program to determine the number of total electrons in the system. By default, the NELECT for a neutral system $\mathrm{Ca}_{2} \mathrm{~N}$ is 9 . The vASP program adds a uniformly distributed negative charge to compensate for the charged $\left[\mathrm{Ca}_{2} \mathrm{~N}\right]^{+}$ model [42]. There, the model system is no longer an electride and shows no electron localization between the positively charged $\left[\mathrm{Ca}_{2} \mathrm{~N}\right]^{+}$layers [Fig. 3(a)]. By taking binary systems as an example, the electron-rich systems can be defined to a formulated general form as $\left[A_{x} B_{y}\right]^{n+}$. $(n e)^{n-}(x, y$, and $n$ are integers, $A$ and $B$ are cationic and anionic elements as electron donors and acceptors, respectively), where these systems possess more electrons than expected from the well-established octet rule by breaking up the standard rules of valency. Thus, these electron-rich ionic systems are different from conventional ionic solids (e.g., $\mathrm{Ca}_{3} \mathrm{~N}_{2}$, $\mathrm{YN}$, and $\mathrm{NaCl}$ ).

The electronegativity of the elements is another critical principle for designing electrides, because it is a direct measure of an elements' ability to attract or donate electrons. We choose metal nitrides (La and Ac, representative of lanthanides and actinides, respectively) and $\mathrm{Ca}_{2} X$ ( $X$ is nonmetallic elements or metallic elements with high electronegativity comparable to nonmetals, such as $\mathrm{Ge}, \mathrm{Sb}$, and $\mathrm{Bi}$ ) as two model systems to illustrate how electronegativity of cationic and anionic elements affects the formation of electrides in certain systems. Metal nitrides containing excess electrons, such as $\left[\left[M_{4} N\right]^{+} \cdot e^{-}\right.$for alkali metal nitrides and $\left[M_{2} N\right]^{+} \cdot e^{-}$for alkaline earth metal nitrides, are examined. The formation probability of electrides, namely, the ratio of the generated electride structures to all structures produced by our inverse design simulations, is plotted in Figs. 3(b) and 3(c). It is seen that only metallic elements with low electronegativity favor electride formation. Specifically, nitrides [Fig. 3(b)] formed by metallic elements in groups IA, IIA, and IIB and Al have higher probabilities of electride formation, whereas the probabilities are much lower for group IVB (e.g., $\mathrm{Zr}$ and Hf) elements. A few electrides in Ti and V nitrides appear; however, they are energetically too unfavorable to be synthesized. Other metallic elements with even higher 
(a)

(b)

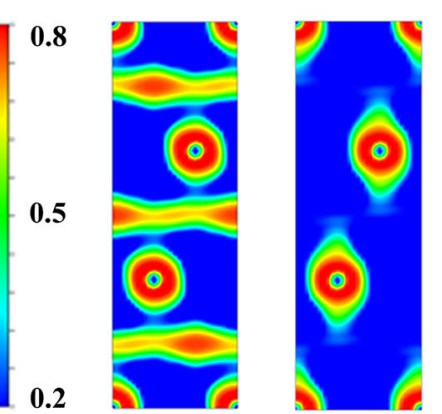

(c)

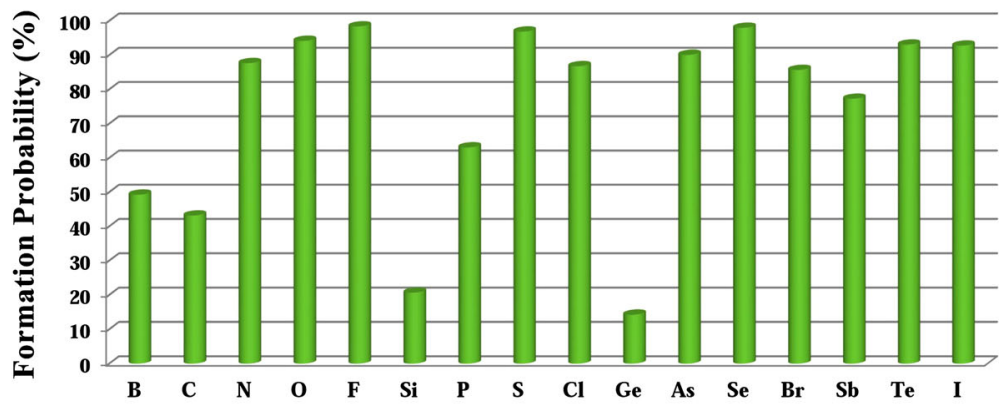

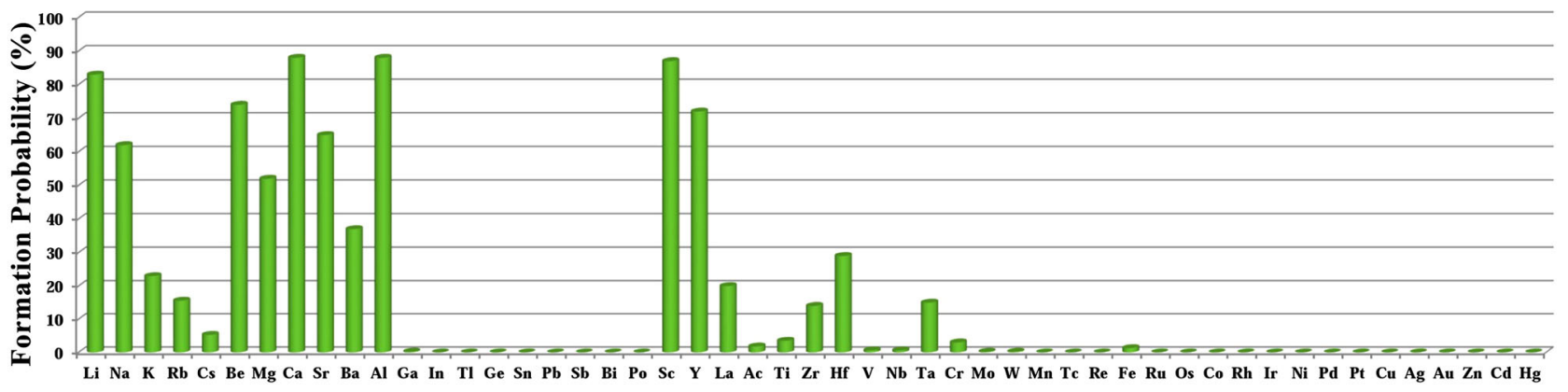

FIG. 3. Tests for the design principles. (a) ELF of $\mathrm{Ca}_{2} \mathrm{~N}$ (left-hand panel) on the $(110)_{R}$ plane parallel to the hexagonal $c$ axis. Upon removal of one excess electron per $\mathrm{Ca}_{2} \mathrm{~N}$, the system loses electron localization (right-hand panel). Formation probabilities of electrides (ratios of electride phases to all structures generated by CALYPSO code in a simulation, characterizing the probability of the system to form electrides) in metal nitrides (b) and $\mathrm{Ca}_{2} X$ compounds (c). Metal and $X$ elements are listed on the $x$ axis and the formation probabilities of electrides are shown on the $y$ axis, respectively. The detailed testing metal nitrides and $\mathrm{Ca}_{2} X$ compounds with their formation probabilities in (b) and (c) are listed in Tables S1 and S2 of Supplemental Material [39].

electronegativity (e.g., $\mathrm{Cr}$ and $\mathrm{Hg}$; bold black frame in Fig. S2 of Ref. [39]) could not form electride phases.

For the $\mathrm{Ca}_{2} X$ systems [Fig. 3(c)], most of the tested objects have a high electride formation probability of more than $50 \%$, except for $\mathrm{Ca}_{2} \mathrm{~B}$ and $\mathrm{Ca}_{2}$ group IVA compounds (e.g., $\mathrm{Ca}_{2} \mathrm{C}, \mathrm{Ca}_{2} \mathrm{Si}$, and $\mathrm{Ca}_{2} \mathrm{Ge}$ ), which are not intrinsically electron-rich compounds. Therefore, most of the generated structures are not electrides. Electride structures do appear, only when the anionic elements form bonding pairs in the structure. With saturation of some unpaired electrons in anionic elements, the structure becomes an electron-rich system suitable for forming an electride.

Comparing the model calculation results of $\mathrm{Ca}_{2} \mathrm{X}$ systems [Fig. 3(c)] with those of metal nitrides [Fig. 3(b)] shows that the choice of cationic elements (rather than the anionic elements) has a critical effect on the formation of electrides. Additionally, based on the testing results of the formation probability shown in Figs. 3(b) and 3(c), it is not unreasonable to establish the no. 2 rule of thumb for design of electrides in compounds containing cationic elements with low electronegativity (e.g., lower than Be, as depicted in Fig. S2 of Supplemental Material [39]).

Through a large number of testing simulations, we are able to establish these two rules of thumb for design of electrides. On the one hand, these rules are empirical, and one cannot rule out the possibility of finding electrides that violate one of these rules, as we have tested on $\mathrm{Ca}_{2} \mathrm{~B}$ and $\mathrm{Ca}_{2}$ group IVA compounds. On the other hand, they can be very efficient to act as a general guide for the design of electrides in a wide range of materials. Accordingly, as we show below, a large number of 89 electron-rich systems composed of cationic elements with low electronegativity are screened to be potential electrides. Furthermore, all known inorganic electrides at ambient conditions (e.g., $\mathrm{C} 12 \mathrm{~A} 7, \mathrm{Y}_{5} \mathrm{Si}_{3}$, and $\mathrm{Ca}_{2} \mathrm{~N}$ with its variants) satisfy these two established rules of thumb.

\section{Electrides identified by inverse-design simulation}

We conduct large-scale computer simulations with the CALYPSO code for 99 binary $A_{2} B$ and $A B$ systems composed of 11 cationic $A$ elements with low electronegativity as electron donors and nine anionic $B$ elements (seven nonmetallic elements and two metallic elements with high electronegativity) as electron acceptors (Fig. 4). These targeted systems satisfy the two rules of thumb on design of inorganic electrides established in this work that contain excess electrons and cationic elements with low electronegativity. Our calculations reproduce the correct anti$\mathrm{CdCl}_{2}$ structure shared by four known electrides, $\mathrm{Ca}_{2} \mathrm{~N}$ [14], $\mathrm{Sr}_{2} \mathrm{~N}, \mathrm{Ba}_{2} \mathrm{~N}$ [27], and $\mathrm{Y}_{2} \mathrm{C}$ [26].

We identify new electride structures in 89 systems, shown in the stability map [Fig. 4(a)] and Fig. S7 of the Supplemental Material. Our simulation generates many electride structures for each system; however, only the electride structure with the lowest energy is shown in 


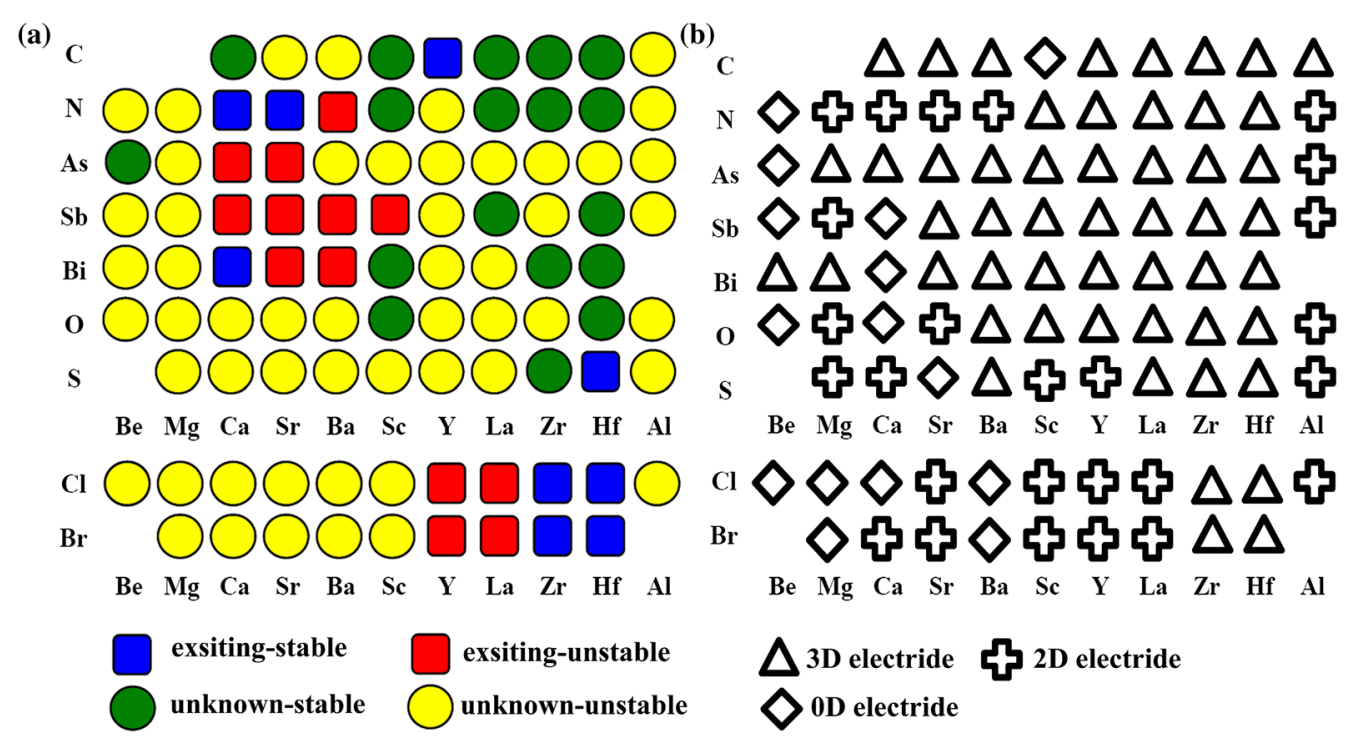

FIG. 4. Inverse-design results for binary electrides. (a) Stability map of $A_{2} B$ (top) and $A B$ (bottom) ( $A$, electron donor; $B$, electron acceptor) electrides. $A$ and $B$ elements are shown in horizontal and vertical directions, respectively. Stable electrides are pointed to the electride structures with negative formation energies in their lowest-energy states, i.e., the thermodynamically most stable states, while metastable electrides are those either not in the lowest-energy states or having positive formation energy. Blue and red squares indicate stable and metastable existing compounds, respectively. Green and yellow circles indicate stable and metastable compounds yet to be synthesized, respectively. (b) Type map of $A_{2} B$ (top) and $A B$ (bottom) electrides. Triangles, crosses, and diamonds indicate 3D, 2D, and OD electrides, respectively.

Fig. 4(a) for taking the consideration of experimental synthesis. The three-dimensional ELF maps to characterize the localized electrons of these lowest-energy electrides are presented in Fig. S4 of the Supplemental Material. All structural information and formation energies of these designed electrides are listed in Table S3 of the Supplemental Material. Of the 89 electrides, 18 are existing compounds that have not been identified as electrides [squares, Fig. 4(a)]. The other 71 electrides [circles, Fig. 4(a)] are unknown compounds that have not been synthesized, of which 18 [green circles, Fig. 4(a)] are thermodynamically stable with negative formation energies in their lowest-energy states and dynamically stable with the absence of any imaginary frequency in phonon spectra (see Fig. S8 of Supplemental Material [39]); the other 53 [yellow circles, Fig. 4(a)] are metastable with positive formation energies or not in their lowest-energy states. The formation energies of the 89 electrides and the relative energies with respect to their ground-state forms are provided in Table S3 of the Supplemental Material [39].

Our target systems contain intrinsic excess electrons that are fundamentally different from conventional ionic solids, explaining why most of the electrides are energetically metastable. In reality, these metastable electrides might be synthesizable, as various metastable electrides have already been synthesized [red squares, Fig. 4(a)]. Furthermore, our inverse design simulations identify a rich variety of previously unknown electride prototype structures. Of 89 new electride structures, there are 33 new prototype structures as listed in Table S3 of the Supplemental
Material [39], of which 19 are not in the known structure databases $[43,44]$.

Based on the localization of interstitial electrons and the distinct conductive properties of the systems, the 89 electrides are classified as 3D, 2D, 0D species [type map, Fig. 4(b)]. There are 52, 22, and 15 inorganic electrides of each type, respectively. We select $\mathrm{Ca}_{2} \mathrm{C}, \mathrm{LaCl}$, and $\mathrm{Be}_{2} \mathrm{~N}$ as typical examples of each to discuss their structural and electronic properties.

\section{E. Structural and electronic properties of designed electrides}

3D electrides.-In 3D electrides [triangles, Fig. 4(b)], excess electrons are partially localized in the interstitial spaces of the lattice. There is a subtle balance between localization and delocalization of excess electrons that contribute to the conductivity in three dimensions. $\mathrm{Ca}_{2} \mathrm{C}$ is typical of the 3D electrides with a body-centered tetragonal $I 4 / \mathrm{mmm}$ structure [right-hand panel, Fig. 5(a)] consisting of $\mathrm{Ca}^{2+}$ and $\mathrm{C}_{2}^{2-}$. This is a new electride structure that is different from the known structures in databases $[43,44]$ with the formation energy of $-2.0 \mathrm{meV} /$ atom, and it can be derived from the insulating $\mathrm{CaC}_{2}$ [45] [left-hand panel, Fig. 5(a)] by replacing the dashed $\mathrm{C}_{2}$ dimers with interstitially localized electrons.

ELF results [right-hand panel, Fig. 5(b)] show that excess electrons are partially localized in cavity sites linked by delocalized electrons. The calculated partial electron density for the region near the Fermi energy [right-hand 


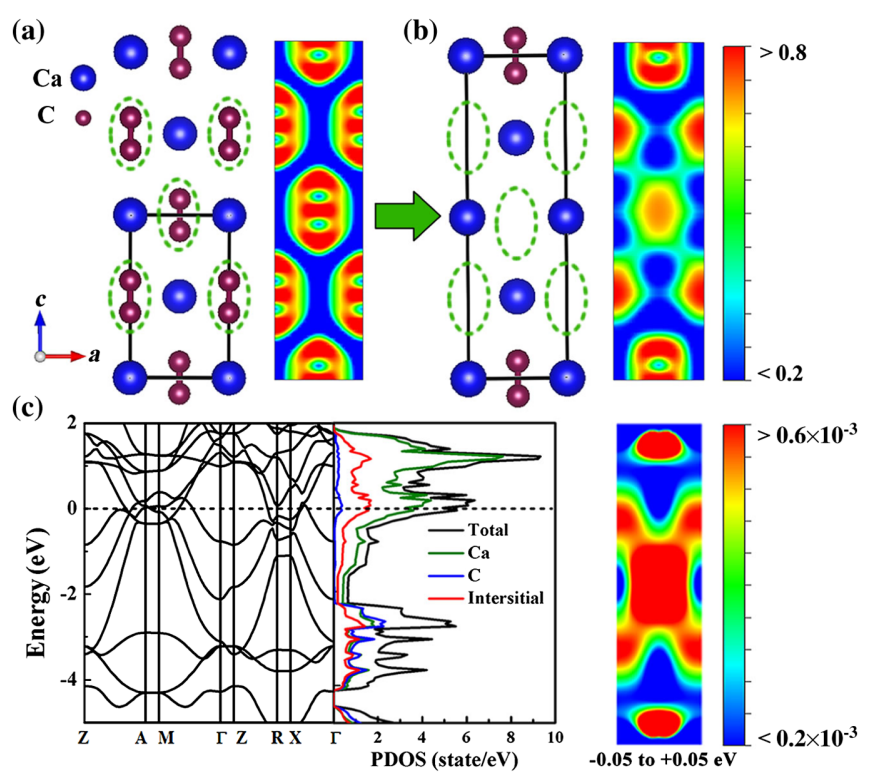

FIG. 5. Structural and electronic properties of $\mathrm{Ca}_{2} \mathrm{C}$ and $\mathrm{CaC}_{2}$. (a) Crystal structure (left-hand panel) and ELF (right-hand panel) on the $(0 \overline{1} 2)_{R}$ plane of $\mathrm{CaC}_{2}$. (b) Crystal structure (left-hand panel) and ELF (right-hand panel) on the $(012)_{R}$ plane of $\mathrm{Ca}_{2} \mathrm{C}$. Excess electrons localize in the interstitial regions indicated by dashed circles. (c) Electronic properties of $\mathrm{Ca}_{2} \mathrm{C}$ : band structure and projected density of states (PDOS) (left-hand panel), and partial electron density for region near $E_{F}(|E|<0.05 \mathrm{eV})$ on the $(012)_{R}$ plane (right-hand panel). The dashed line indicates the Fermi energy $\left(E_{F}\right)$.

panel, Fig. 5(c)] shows that these delocalized electrons dominate the conductivity of $\mathrm{Ca}_{2} \mathrm{C}$. The $3 \mathrm{D}$ conducting behavior of excess electrons could also be inferred from the band structure of $\mathrm{Ca}_{2} \mathrm{C}$ [left-hand panel, Fig. 5(c)] at the Fermi level that crosses over the high-symmetry directions along $Z-A, A-M$, and $X-\Gamma$.

$2 D$ electrides. - In 2D electrides [crosses, Fig. 4(b)], the excess electrons are confined to the interspaces between cationic layers and contribute to the anisotropic conductivity of the system. Localized electrons in 2D electrides are not evenly distributed and there are non-nuclear electron maxima at crystallographic positions. 2D electrides usually have layered structures; however, not all layered electrides fall into this category. For example, although $\mathrm{Sc}_{2} \mathrm{~N}$ and $\mathrm{Y}_{2} \mathrm{~N}$ share the same layered structure as $\mathrm{Ca}_{2} \mathrm{~N}$, they are not $2 \mathrm{D}$ but $\mathrm{OD}$ electrides because the excess electrons are highly localized at the interstitial crystallographic sites and do not contribute to the conductivity.

$\mathrm{LaCl}$ [46] has a layered $R-3 m$ structure with $A B C B A$ atomic packing ( $A, B$, and $C$ are $\mathrm{Cl}^{-}$sheets, $\mathrm{La}^{3+}$ sheets, and anionic electron layers, respectively) [Fig. 6(a)]. Both La and $\mathrm{Cl}$ atoms form a trigonal arrangement in their sheets. The ELF results [top panel, Fig. 6(b)] show that the excess electrons are confined to the interlayer interstitial spaces between two La sheets (white dashed circles). The three centers of the maximally localized regions are arranged

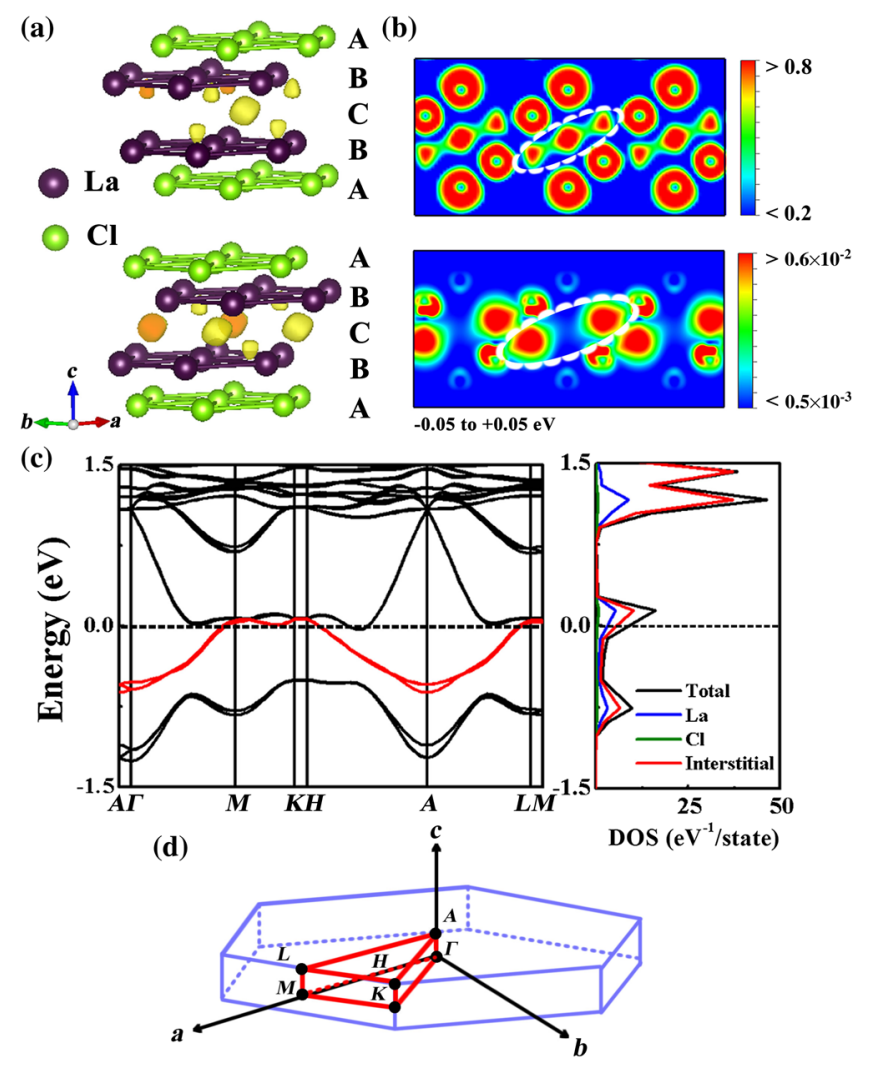

FIG. 6. Structural and electronic properties of LaCl. (a) 3D ELF of $\mathrm{LaCl}$ with an isosurface value of 0.7. (b) $2 \mathrm{D} \mathrm{ELF}$ (top panel) on the $\left(\begin{array}{lll}\overline{1} & 1 & 0\end{array}\right)_{R}$ plane and partial electron density for the region near $E_{F}(|E|<0.05 \mathrm{eV})$ (bottom panel) on the $\left(\begin{array}{lll}\overline{1} & \overline{1} & 0\end{array}\right)_{R}$ plane. (c) Band structure and electron density of state of $\mathrm{LaCl}$. The dashed line indicates the Fermi energy $\left(E_{F}\right)$. (d) The high-symmetry lines (red) are in the first Brillouin zone.

into groups that are connected throughout the layer by delocalized electrons. The conducting behaviors of the three center excess electrons in one group are different. The partial electron density for the region near the Fermi energy indicates that the excess electrons at both ends of one group [dashed white line, bottom panel, Fig. 6(b)] are partially localized and contribute to the conductivity of the system; however, the excess electrons at the center of the group are well localized and do not show conducting behavior. The dispersive band near the Fermi energy [red, left-hand panel, Fig. 6(c)] is mainly occupied by interstitial electrons, as shown by the density of state [right-hand panel, Fig. 6(c)]. The high-symmetry lines of $\Gamma-M-K-\Gamma$ and $A-L-H-A$ in the reciprocal space in Fig. 6(d) indicate the 2D planes of the structure in real space where the electrons are localized. The red bands cross the Fermi energy only along these symmetric lines, indicating 2D anisotropic conducting behavior in the system.

$O D$ electrides.-Electron localization of OD electrides [diamonds, Fig. 4(b)] is geometrically similar to that of 3D electrides. However, excess electrons in OD electrides are completely localized and they do not contribute to 


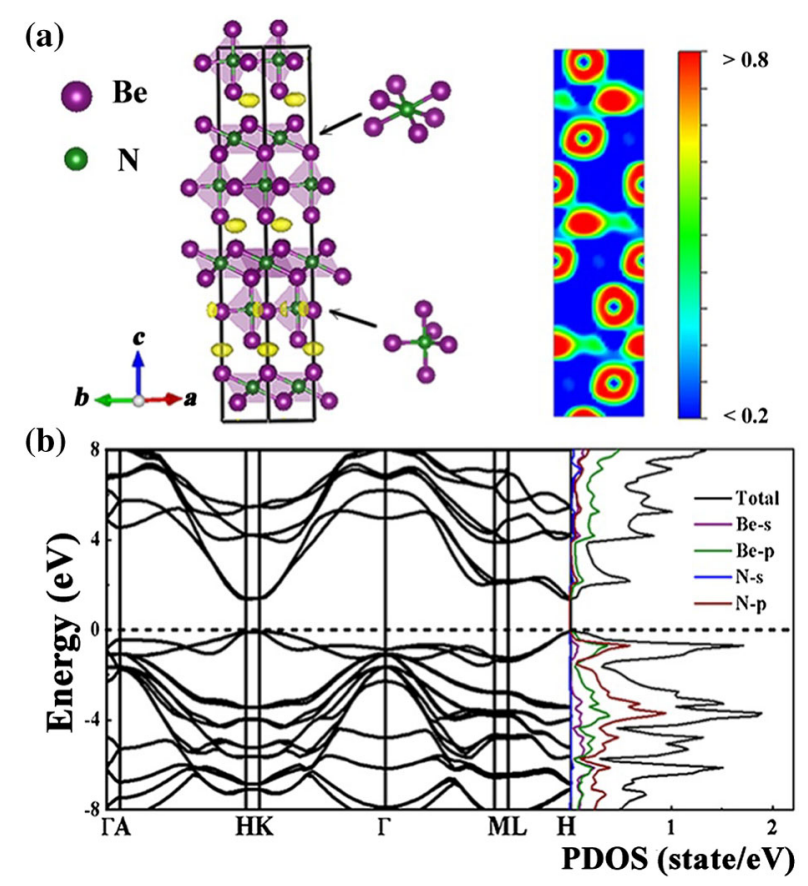

FIG. 7. Semiconducting $\mathrm{Be}_{2} \mathrm{~N}$ as a $0 \mathrm{D}$ electride. (a) $3 \mathrm{D}$ ELF of $\mathrm{Be}_{2} \mathrm{~N}$ (left-hand panel) with an isosurface value of 0.83 . Sixfold and fivefold coordinated $\mathrm{Be}$ atoms are shown as structural units in the left-hand panel. The 2D ELF on the $(110)_{R}$ plane in the righthand panel shows the isolated interstitial localization regions consistent with the 3D ELF. (b) Band structure and PDOS of $\mathrm{Be}_{2} \mathrm{~N}$. The HSE06 functional $[47,48]$ corrects the DFT band gap of $1.51 \mathrm{eV}$ to $1.96 \mathrm{eV}$. The dashed line indicates the Fermi energy $\left(E_{F}\right)$.

the conductivity. Thus, OD electrides typically show semiconducting or insulating properties, which are similar to some known high-pressure electrides, such as transparent $h P 4 \mathrm{Na}$ at $320 \mathrm{GPa}[17]$ and semiconducting Aba2 - $40 \mathrm{Li}$ at $70 \mathrm{GPa}$ [22].

$\mathrm{Be}_{2} \mathrm{~N}$ adopts an $R 3 \mathrm{~m}$ rhombohedral metastable structure with the positive formation energy of $+0.189 \mathrm{eV} /$ atom, a new electride structure that is also not in the existing structure database $[43,44]$. There are two kinds of $\mathrm{N}$ atoms in $\mathrm{Be}_{2} \mathrm{~N}$ : (i) $\mathrm{N}$ atoms that are sixfold coordinated with $\mathrm{Be}$ atoms forming faces sharing octahedrons along the $x-y$ plane and (ii) $\mathrm{N}$ atoms that are fivefold coordinated with $\mathrm{Be}$ atoms forming $\mathrm{N}$ centered hexahedrons [left-hand panel, Fig. 7(a)]. Each hexahedron links to its neighboring hexahedrons or octahedrons by sharing one Be atom. The ELF results [Fig. 7(a)] show that excess electrons are entirely localized in empty crystallographic positions [yellow spheres, left-hand panel, Fig. 7(a)] in the interstitial. The localized electrons are discrete, leading to the semiconductivity of the system, as shown by its band structure [Fig. 7(b)].

\section{DISCUSSION}

Our inverse-design scheme has the advantage of searching for the optimum electride structures that give the highest electron localization possible. Moreover, our method can identify richer electride structures than the energy-based traditional searching method does. By taking $\mathrm{La}_{2} \mathrm{C}$ as an example (see Fig. S5 of Supplemental Material [39]), our method generates 216 electride structures out of 900 structures, where the highest $\omega$ value reaches $2.19 \%$. However, in the traditional searching method, only 156 electride structures are generated, with the highest $\omega$ reaching a much smaller value of $1.21 \%$. For the 89 newly designed electrides, their optimum electride structures (structure details are listed in Table S4 of Supplemental Material [39]) are high in energy, but they may be used as good prototype structures for future design of electrides in other material systems through, for example, high-throughput simulations.

We extend electrides into general electron-rich forms [such as $\left[A_{x} B_{y}\right]^{n+} \cdot(n e)^{n-}$ for binary systems] by breaking up the standard rules of valency in conventional ionic compounds. Stoichiometries $A_{2} B$ and $A B$ are used in this work as representative examples; there are many alternative candidate stoichiometries in other binary, ternary, quaternary, and more complex compounds that are ripe for exploration. Although currently there are several known inorganic electrides and their practical applications are limited, we believe the situation can be substantially changed with the aid of our inverse-design method. The findings of inorganic electrides may become a routine activity.

We use electron-rich ionic systems in our simulations; however, this does not exclude the possibility of finding electrides in conventional ionic compounds. There, electrides could be identified where specific covalent bonds are formed. For example, $\mathrm{Ca}_{2} \mathrm{C}$ is placed into an electride system when $\mathrm{C}$ atoms form pairs.

In view of the observed superconductivity in the heavily electron-doped mayenite $[49,50]$, we expect that some of our predicted conducting electrides might also show similar superconducting properties. Through explicit calculations of electron-phonon interaction, we find that some $3 \mathrm{D}$ electrides could be superconducting with low calculated $T_{c}$ values (e.g., $5 \mathrm{~K}$ for $\mathrm{Ca}_{2} \mathrm{Bi}$ and $4.17 \mathrm{~K}$ for $\mathrm{Sc}_{2} \mathrm{As}$ ). The interstitial localized electrons might play an important role in the superconductivity of these compounds, and further study of the mechanism is required.

\section{METHODS}

Structure prediction.-Our structure searching simulations are performed by the swarm intelligence CALYPSO method $[29,30,40]$ enabling global minimization of energy surfaces by merging $a b$ initio total-energy calculations, as implemented in the CALYPSO code. The method is benchmarked on various known systems [51-53]. The degree of interstitial electron localization is introduced as the global variable function in searching for electrides. 
First-principles calculations.-All the electronic structure calculations are performed with the density-functional theory within the Perdew-Burke-Ernzerhof functional of the generalized gradient approximation as implemented in the Vienna ab initio simulation package (VASP) [54]. The projector-augmented wave [55] method is adopted with the projector-augmented wave potentials taken from the VASP library. The HSE06 hybrid functional $[47,48]$ reproduces the band gap of semiconductors well, and is used to revise the band gaps [56] of the semiconducting phases of OD electrides calculated by the Perdew-Burke-Ernzerhof functional. The plane-wave kinetic energy cutoffs of $800 \mathrm{eV}$ and Monkhorst-Pack Brillouin zone sampling grid with a resolution of $2 \pi \times 0.03 \AA^{-1}$ are chosen to ensure that all the enthalpy calculations are well converged to better than $1 \mathrm{meV} /$ atom. We note that DFT calculations tend to favor overly delocalized electrons due to the many-electron selfinteraction error [57,58], which may lead to misidentify electrides. However, so far, the DFT calculations correctly reproduce all known inorganic electrides. Phonon frequencies are calculated by using a supercell approach [59], as implemented in the PHONOPY code [60]. Phonon dispersion and electron-phonon coupling (EPC) calculations are performed within the framework of the linear-response theory by using QUANTUM-ESPRESSO [61]. Norm-conserving pseudopotentials for $\mathrm{Ca}, \mathrm{Sb}, \mathrm{Sc}$, and $\mathrm{As}$ are used with kinetic energy cutoffs of 80 Ry. $3 \times 3 \times 3$ and $4 \times 4 \times 4 q$ meshes in the first Brillouin zones are adopted in the EPC calculations for $\mathrm{Ca}_{2} \mathrm{Sb}$ and $\mathrm{Sc}_{2} \mathrm{As}$, respectively. Monkhorst-Pack grids of $12 \times 12 \times 12$ and $16 \times 16 \times 8$ are used to ensure $k$-point sampling convergence with Gaussians of width 0.05 Ry for $\mathrm{Ca}_{2} \mathrm{Sb}$ and $\mathrm{Sc}_{2} \mathrm{As}$, respectively, in order to approximate the zero-width limit in the calculations of the EPC parameter, $\lambda$. It should be mentioned that since the screened Coulomb repulsion parameter $\mu^{*}$ (in the Allen-Dynes modified McMillan equation) cannot be evaluated by any firstprinciples method, our theoretically predicted $T_{c}$ has an intrinsic uncertainty originating from the empirical choice of $\mu^{*}$. Depending on particular materials, $\mu^{*}$ usually falls in the range between 0.1 and 0.2 . In our study, a typical value for $\mu^{*}$ of 0.1 is used in predicting $T_{c}$ values.

\section{ACKNOWLEDGMENTS}

Y.Z., Y. W., and Y.M. acknowledge funding from the National Natural Science Foundation of China under Grants No. 11274136, No. 11404128, and No. 11534003; the National Key Research and Development Program of China under Grant No. 2016YFB0201200; and the 2012 Changjiang Scholars Program of China. L. Z. acknowledges funding support from the Recruitment Program of Global Experts (the Thousand Young Talents Plan). H. W. acknowledges funding support from the 2013 Program for New Century Excellent Talents in University. Y. M. designed the research. Y. Z. performed most of the calculations. Y. Z., H. $\mathrm{W}$., and Y.W. coded the inverse design method into
CALYPSO. Y.Z. and Y. M. analyzed the results and wrote the manuscript. All authors commented on the manuscript. We thank H. Liu for helpful discussions and comments. Part of the calculation was performed in the high performance computing center of Jilin University and at Tianhe2-JK in the Beijing Computational Science Research Center.

[1] A. Walsh and D. O. Scanlon, Electron Excess in Alkaline Earth Sub-Nitrides: 2D Electron Gas or 3D Electride?, J. Mater. Chem. C 1, 3525 (2013).

[2] J. L. Dye and M. G. DeBacker, Physical and Chemical Properties of Alkalides and Electrides, Annu. Rev. Phys. Chem. 38, 271 (1987).

[3] M. Y. Redko, J. E. Jackson, R. H. Huang, and J. L. Dye, Design and Synthesis of a Thermally Stable Organic Electride, J. Am. Chem. Soc. 127, 12416 (2005).

[4] A. Ellaboudy, J. L. Dye, E. Lansing, and P. B. Smith, Cesium 18-Crown-6 Compounds. A Crystalline Ceside and a Crystalline Electride, J. Am. Chem. Soc. 105, 6490 (1983).

[5] S. B. Dawes, D. L. Ward, R. H. Huang, and J. L. Dye, First Electride Crystal Structure, J. Am. Chem. Soc. 108, 3534 (1986).

[6] J. Kim, J. L. Eglin, A. S. Ellaboudy, L. E. H. Mcmills, S. Huang, and J. L. Dye, ${ }^{87} \mathrm{Rb},{ }^{85} \mathrm{Rb}$, and ${ }^{39} \mathrm{~K}$ NMR Studies of Alkalides, Electrides, and Related Compounds, J. Phys. Chem. 100, 2885 (1996).

[7] A. S. Ichimura, J. L. Dye, M. A. Camblor, and L. A. Villaescusa, Toward Inorganic Electrides, J. Am. Chem. Soc. 124, 1170 (2002).

[8] J. L. Dye, Electrides: Early Examples of Quantum Confinement, Acc. Chem. Res. 42, 1564 (2009).

[9] R. C. Phillips, W. P. Pratt, and J. L. Dye, Thermionic Emission from Cold Electride Films, Chem. Mater. 12, 3642 (2000).

[10] S. Matsuishi, Y. Toda, M. Miyakawa, K. Hayashi, T. Kamiya, M. Hirano, I. Tanaka, and H. Hosono, HighDensity Electron Anions in a Nanoporous Single Crystal: $\left[\mathrm{Ca}_{24} \mathrm{Al}_{28} \mathrm{O}_{64}\right]^{4+}\left(4 e^{-}\right)$, Science 301, 626 (2003).

[11] Y. Toda, S. Matsuishi, K. Hayashi, K. Ueda, T. Kamiya, M. Hirano, and H. Hosono, Field Emission of Electron Anions Clathrated in Subnanometer-Sized Cages in $\left[\mathrm{Ca}_{24} \mathrm{Al}_{28} \mathrm{O}_{64}\right]^{4+}\left(4 e^{-}\right)$, Adv. Mater. 16, 685 (2004).

[12] M. Tsai, U. Seip, I. C. Bassignana, J. Kuppers, and G. Ertl, Vibrational Spectroscopy Study on the Interaction of $\mathrm{N}_{2}$ with Clean and K-Promoted Fe (111) Surfaces: $\pi$-Bonded Dinitrogen as Precursor for Dissociation, Surf. Sci. 155, 387 (1985).

[13] M. Kitano, Y. Inoue, Y. Yamazaki, F. Hayashi, S. Kanbara, S. Matsuishi, T. Yokoyama, S. W. Kim, M. Hara, and H. Hosono, Ammonia Synthesis Using a Stable Electride as an Electron Donor and Reversible Hydrogen Store, Nat. Chem. 4, 934 (2012).

[14] K. Lee, S. W. Kim, Y. Toda, S. Matsuishi, and H. Hosono, Dicalcium Nitride as a Two-Dimensional Electride with an Anionic Electron Layer, Nature (London) 494, 336 (2013). 
[15] Y. J. Kim, S. M. Kim, E. J. Cho, H. Hosono, J. W. Yang, and S. W. Kim, Two-Dimensional Inorganic Electride-Promoted Electron Transfer Efficiency in Transfer Hydrogenation of Alkynes and Alkenes, Chem. Sci. 6, 3577 (2015).

[16] S. Zhao, Z. Li, and J. Yang, Obtaining Two-Dimensional Electron Gas in Free Space without Resorting to Electron Doping: An Electride Based Design, J. Am. Chem. Soc. 136, 13313 (2014).

[17] Y. Ma, M. Eremets, A. R. Oganov, Y. Xie, I. Trojan, S. Medvedev, A. O. Lyakhov, M. Valle, and V. Prakapenka, Obtaining Two-Dimensional Electron Gas in Free Space without Resorting to Electron Doping: An Electride Based Design, Nature (London) 458, 182 (2009).

[18] A. R. Oganov, Y. Xu, Y. Ma, I. Errea, A. Bergara, and A. O. Lyakhov, Exotic Behavior and Crystal Structures of Calcium Under Pressure, Proc. Natl. Acad. Sci. U.S.A. 107, 7646 (2010).

[19] M. Miao and R. Hoffmann, High Pressure Electrides: The Chemical Nature of Interstitial Quasi-Atoms, J. Am. Chem. Soc. 137, 3631 (2015).

[20] P. Li, G. Gao, Y. Wang, and Y. Ma, Crystal Structures and Exotic Behavior of Magnesium Under Pressure, J. Phys. Chem. C 114, 21745 (2010).

[21] C. J. Pickard and R. J. Needs, Aluminum at Terapascal Pressures, Nat. Mater. 9, 624 (2010).

[22] J. Lv, Y. Wang, L. Zhu, and Y. Ma, Predicted Novel HighPressure Phases of Lithium, Phys. Rev. Lett. 106, 015503 (2011).

[23] M. Miao and R. Hoffmann, High Pressure Electrides: A Predictive Chemical and Physical Theory, Acc. Chem. Res. 47, 1311 (2014).

[24] T. Tada, S. Takemoto, S. Matsuishi, and H. Hosono, HighThroughput Ab Initio Screening for Two-Dimensional Electride Materials, Inorg. Chem. 53, 10347 (2014).

[25] T. Inoshita, S. Jeong, N. Hamada, and H. Hosono, Exploration for Two-Dimensional Electrides via Database Screening and Ab Initio Calculation, Phys. Rev. X 4, 031023 (2014).

[26] X. Zhang, Z. Xiao, H. Lei, Y. Toda, S. Matsuishi, T. Kamiya, S. Ueda, and H. Hosono, Two-Dimensional Transition-Metal Electride $\mathrm{Y}_{2} \mathrm{C}$, Chem. Mater. 26, 6638 (2014).

[27] O. Reckeweg and F. J. Disalvo, Alkaline Earth Metal Nitride Compounds with the Composition $M_{2} \mathrm{~N} X(M=\mathrm{Ca}, \mathrm{Sr}, \mathrm{Ba}$; $X=\mathrm{H}, \mathrm{Cl}$ or $\mathrm{Br}$ ), Solid State Sci. 4, 575 (2002).

[28] Y. Lu, J. Li, T. Tada, Y. Toda, S. Ueda, T. Yokoyama, M. Kitano, and $\mathrm{H}$. Hosono, Water Durable Electride $\mathrm{Y}_{5} \mathrm{Si}_{3}$ : Electronic Structure and Catalytic Activity for Ammonia Synthesis, J. Am. Chem. Soc. 138, 3970 (2016).

[29] Y. Wang, J. Lv, L. Zhu, and Y. Ma, Crystal Structure Prediction via Particle-Swarm Optimization, Phys. Rev. B 82, 094116 (2010).

[30] Y. Wang, J. Lv, L. Zhu, and Y. Ma, CALYPSO: A Method for Crystal Structure Prediction, Comput. Phys. Commun. 183, 2063 (2012).

[31] X. Zhang, Y. Wang, J. Lv, Q. Li, C. Zhu, Q. Li, M. Zhang, and Y. Ma, First-Principles Structural Design of Superhard Materials, J. Chem. Phys. 138, 114101 (2013).

[32] A. D. Becke and K. E. Edgecombe, A Simple Measure of Electron Localization in Atomic and Molecular Systems, J. Chem. Phys. 92, 5397 (1990).
[33] A. Savin, R. Nesper, S. Wengert, and T. E. Fassler, ELF: The Electron Localization Function, Angew. Chem., Int. Ed. Engl. 36, 1808 (1997).

[34] J. K. Burdett and T. A. McCormick, Electron Localization in Molecules and Solids: The Meaning of ELF, J. Phys. Chem. A 102, 6366 (1998).

[35] Y. Wang, M. Miao, J. Lv, L. Zhu, K. Yin, H. Liu, and Y. Ma, An Effective Structure Prediction Method for Layered Materials Based on 2D Particle Swarm Optimization Algorithm, J. Chem. Phys. 137, 224108 (2012).

[36] Z. Li, J. Yang, J. G. Hou, and Q. Zhu, Is Mayenite without Clathrated Oxygen an Inorganic Electride?, Angew. Chem., Int. Ed. Engl. 43, 6479 (2004).

[37] A. Savin, The Electron Localization Function (ELF) and Its Relatives: Interpretations and Difficulties, J. Mol. Struc. Theochem. 727, 644 (2005).

[38] I. Vidal, S. Melchor, and J. A. Dobado, On the Nature of Metal-Carbon Bonding: AIM and ELF Analyses of $\mathrm{MCH}_{n}$ ( $n=1-3)$ Compounds Containing Early Transition Metals, J. Phys. Chem. A 109, 7500 (2005).

[39] See Supplemental Material at http://link.aps.org/ supplemental/10.1103/PhysRevX.7.011017 for detailed descriptions and testing results of our inverse-design method, crystal information of 89 newly designed electrides, phonon spectra of 18 stable electrides and other potential prototype structures of electrides.

[40] L. Pauling, The Nature of the Chemical Bond and the Structure of Molecules and Crystals: An Introduction to Modern Structural Chemistry, Vol. 18 (Cornell University Press, Ithaca, NY, 1960).

[41] J. Lv, Y. Wang, L. Zhu, and Y. Ma, Particle-Swarm Structure Prediction on Clusters, J. Chem. Phys. 137, 084104 (2012).

[42] The VASP program adds a uniformly distributed negative charge to compensate for the charged $\left[\mathrm{Ca}_{2} \mathrm{~N}\right]^{+}$model, https://cms.mpi.univie.ac.at/vasp/vasp/NELECT.html.

[43] S. Gražulis, A. Daškevič, A. Merkys, D. Chateigner, L. Lutterotti, M. Quirós, N. R. Serebryanaya, P. Moeck, R. T. Downs, and A. Le Bail, Crystallography Open Database (COD): An Open-Access Collection of Crystal Structures and Platform for World-Wide Collaboration, Nucleic Acids Res. 40, D420 (2012).

[44] Y. Xu, M. Yamazaki, and P. Villars, Inorganic Materials Database for Exploring the Nature of Material, Jpn. J. Appl. Phys. 50, 11RH02 (2011).

[45] G. v. Strackelberg, Die Krystallstruktur des $\mathrm{CaC}_{2}$, Naturwissenschaften 18, 305 (1930).

[46] R. E. Araujo and J. D. Corbett, Lanthanum Monochloride and Lanthanum Sesquichloride, Inorg. Chem. 20, 3082 (1981).

[47] J. Heyd, G. E. Scuseria, and M. Ernzerhof, Hybrid Functionals Based on a Screened Coulomb Potential, J. Chem. Phys. 118, 8207 (2003).

[48] A. V. Krukau, O. A. Vydrov, A. F. Izmaylov, and G. E. Scuseria, Influence of the Exchange Screening Parameter on the Performance of Screened Hybrid Functionals, J. Chem. Phys. 125, 224106 (2006).

[49] M. Miyakawa, S. W. Kim, M. Hirano, Y. Kohama, H. Kawaji, T. Atake, H. Ikegami, K. Kono, and H. Hosono, Superconductivity in an Inorganic Electride $12 \mathrm{CaO} \cdot 7 \mathrm{Al}_{2} \mathrm{O}_{3}: \mathrm{e}^{-}$, J. Am. Chem. Soc. 129, 7270 (2007). 
[50] H. Hosono, S. Kim, S. Matsuishi, S. Tanaka, A. Miyake, K. Shimizu, and S. Kim, Superconductivity in Room-Temperature Stable Electride and High-Pressure Phases of Alkali Metals, Phil. Trans. R. Soc. A 373, 20140450 (2015).

[51] L. Zhu, H. Liu, C. J. Pickard, G. Zou, and Y. Ma, Reactions of Xenon with Iron and Nickel are Predicted in the Earth's Inner Core, Nat. Chem. 6, 644 (2014).

[52] H. Wang, J. S. Tse, K. Tanaka, T. Iitaka, and Y. Ma, Superconductive Sodalite-like Clathrate Calcium Hydride at High Pressures, Proc. Natl. Acad. Sci. U.S.A. 109, 6463 (2012).

[53] Y. Li, J. Hao, H. Liu, Y. Li, and Y. Ma, The Metallization and Superconductivity of Dense Hydrogen Sulfide, J. Chem. Phys. 140, 174712 (2014).

[54] G. Kresse and J. Furthmüller, Efficiency of Ab-Initio Total Energy Calculations for Metals and Semiconductors Using a Plane-Wave Basis Set, Comput. Mater. Sci. 6, 15 (1996).

[55] G. Kresse and D. Joubert, From Ultrasoft Pseudopotentials to the Projector Augmented-Wave Method, Phys. Rev. B 59, 1758 (1999).
[56] J. Heyd, J. E. Peralta, G. E. Scuseria, and R. L. Martin, Energy Band Gaps and Lattice Parameters Evaluated with the Heyd-Scuseria-Ernzerhof Screened Hybrid Functional, J. Chem. Phys. 123, 174101 (2005).

[57] J. P. Perdew and A. Zunger, Self-Interaction Correction to Density-Functional Approximations for Many-Electron Systems, Phys. Rev. B 23, 5048 (1981).

[58] P. Mori-Sánchez, A. J. Cohen, and W. Yang, From Ultrasoft Pseudopotentials to the Projector Augmented-Wave Method, J. Chem. Phys. 125, 1758 (2006).

[59] K. Parlinski, Z. Q. Li, and Y. Kawazoe, First-Principles Determination of the Soft Mode in Cubic $\mathrm{ZrO}_{2}$, Phys. Rev. Lett. 78, 4063 (1997).

[60] A. Togo, F. Oba, and I. Tanaka, First-Principles Calculations of the Ferroelastic Transition between Rutile-Type and $\mathrm{CaCl}_{2}-$ Type $\mathrm{SiO}_{2}$ at High Pressures, Phys. Rev. B 78, 134106 (2008).

[61] S. Scandolo, P. Giannozzi, C. Cavazzoni, S. De Gironcoli, A. Pasquarello, and S. Baroni, First-Principles Codes for Computational Crystallography in the QUANTUM-ESPRESSO Package, Z. Kristallogr. 220, 574 (2005). 